\title{
Biomedical Application of Soft X-ray Microscopy with Special Reference to Spectromicroscopy
}

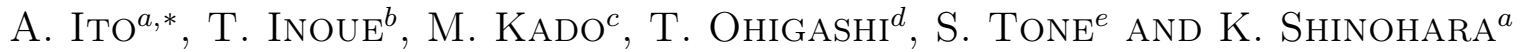 \\ ${ }^{a}$ School of Engineering, Tokai University, Hiratsuka 259-1292, Japan \\ ${ }^{b}$ Kao Corporation, Odawara, Kanagawa 250-0002, Japan \\ ${ }^{c}$ Japan Atomic Energy Agency, Kizugawa 619-0215, Japan \\ ${ }^{d}$ UVSOR Facility, Institute for Molecular Science, Okazaki 444-8585, Japan \\ ${ }^{e}$ Department of Biochemistry, Kawasaki Medical School, Kurashiki 701-0192, Japan
}

\begin{abstract}
Soft X-ray microscopy has great advantages over other types of microscopy on the observation of hydrated specimens at high spatial resolution below $100 \mathrm{~nm}$, and on the elemental and molecular mapping using absorption characteristics. These advantages are particularly suitable for biomedical applications. A possible problem associated with observation of specimens at high spatial resolution in a hydrated environment is described and a strategy to overcome the problem is presented. The latter part of the present report deals with spectromicroscopy to obtain molecular distribution in biological specimens. DNA and protein distributions in isolated nuclei are examined following to the progression of apoptosis at high resolution, and the oxidation product is visualized in human hair in relation to calcium content.
\end{abstract}

DOI: 10.12693/APhysPolA.129.260

PACS: 87.59.--e

\section{Introduction}

The potential advantages of soft X-ray microscopy with X-ray energies below several $\mathrm{keV}$ [1] in biology over conventional microscopy such as light and electron microscopy can be summarized as follows: (1) High spatial resolution imaging in the natural physiological state, particularly using X-rays with photon energies in the "water window" region between $K$ absorption edges of carbon $(284 \mathrm{eV})$ and oxygen $(544 \mathrm{eV}),(2)$ capability of computed tomography (CT) imaging to observe thick specimens, and (3) mapping of elements and molecules using spectromicroscopy for characteristic elements or specific molecules.

Comparing with hard X-ray microscopy which is being actively developed due to its large penetration depth covering tissues, soft X-ray microscopy has remarkable features including achievement of higher spatial resolution at absorption contrast better than $50 \mathrm{~nm}$ or even down to $10 \mathrm{~nm}$. The absorption contrast gives direct information about electron density and is easy to interpret without any image reconstruction processes definitely required for phase contrast imaging. Thus soft X-ray microscopy would be suitable for high resolution observation of a whole cell or intracellular molecular assembly in a thickness of a few $\mu \mathrm{m}$.

On the observation of elemental and molecular map, soft X-ray spectromicroscopy has still several advantages over electron microscopy. Electron-probe microanalysis

\footnotetext{
* corresponding author; e-mail aeito@keyaki.cc.u-tokai.ac.jp
}

(EPMA) or electron energy loss spectroscopy (EELS) for such purposes causes much more radiation damage on the specimens due to the presence of optically forbidden transition and the production of secondary electrons. Energy resolution in chemical mapping is also advantageous for soft X-ray spectromicroscopy. The energy resolution of EELS is around $20 \mathrm{eV}$, while in the case of the soft $\mathrm{X}$ ray spectromicroscopy the energy resolution below $1 \mathrm{eV}$ is achievable depending on a monochromator.

\section{Observation of hydrated specimens}

In order to achieve high spatial resolution in water environment, it is necessary to avoid image blurring caused by Brownian motion. We estimated the degree of blurring for freely diffusible particles as a function of imaging time [2]. This estimate is an extreme case and may occasionally be overestimated, but we could know the upper limit for the required imaging time. According to our calculation, if we need $50 \mathrm{~nm}$ in spatial resolution for example, imaging should be completed within $0.5 \mathrm{~ms}$. In addition, we have to consider X-ray dose to achieve a required spatial resolution. Sayre et al. proposed a method to estimate radiation dose as a function of required spatial resolution [2]. We applied their method to a model nucleoprotein, a complex of DNA and its binding protein histone, with a thickness of $1 \mu \mathrm{m}$, which is surrounded by medium of water containing protein with a ratio of 9 to 1 . Under this condition, we calculated photon fluence in the order of $10^{13}$ photons $/ \mathrm{cm}^{2}$ to achieve $100 \mathrm{~nm}$ spatial resolution. Incidentally, in the same situation the absorbed dose required for electron microscope was estimated about $10^{2}$ times larger than $\mathrm{X}$-ray microscope [3]. To meet these requirements, laser plasma X-rays produced with highly intense laser pulse have been applied 
to capture X-ray image of hydrated specimens in a very short time. A thin-foiled gold target has been irradiated with an intense pulsed laser with $20 \mathrm{~J}$ energy in $600 \mathrm{ps}$ pulse duration to produce laser plasma. The photon flux of the laser-plasma X-ray source in the water window $(2.3-4.4 \mathrm{~nm})$ has been measured to be $1.4 \times 10^{15}$ photons/pulse/sr and it makes possible to capture a soft Xray image of live hydrated biological cells with a single pulse exposure.

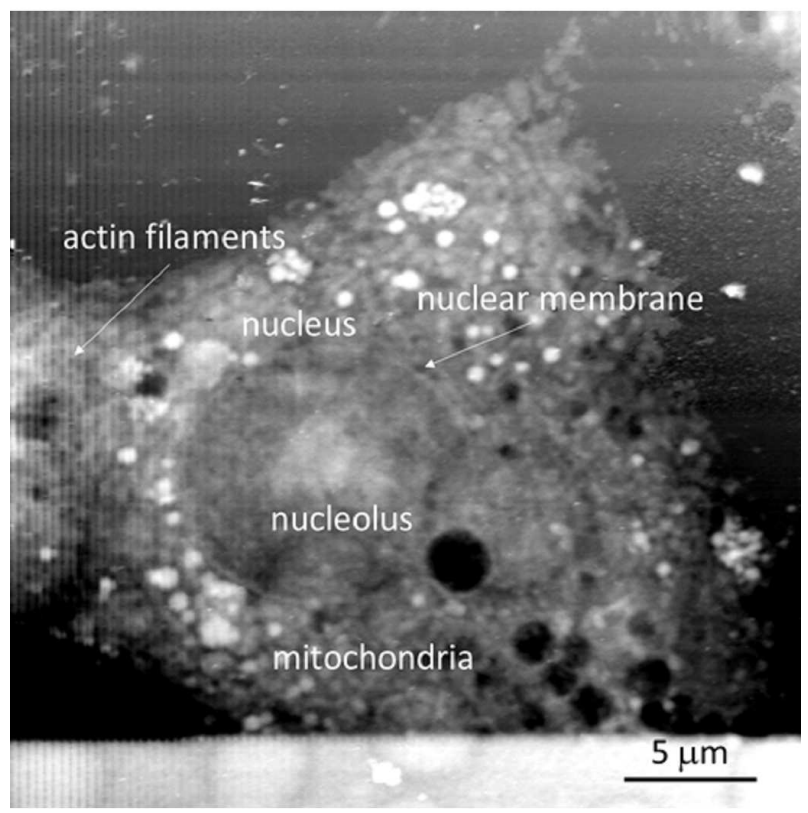

Fig. 1. Soft X-ray images of live hydrated Leydig cells of mouse testis taken with laser plasma X-rays.

Figure 1 shows a recent observation of live mammalian cells, which was achieved below $100 \mathrm{~nm}$ spatial resolution at the single exposure with time duration of $600 \mathrm{ps}$ using a contact microscopy. In Fig. 1, intracellular structures such as chromatin, mitochondria and actin filaments could be clearly identified [4]. Interestingly dense white particle-like structures were identified as oil droplets relating to steroid hormone. Alternative to it, a rapid freezing method frequently used to maintain hydrated structure could be suitable for CT imaging. Such effort has been continued at a beamline dedicated for soft X-ray microscopy, e.g. NCXT at ALS [5].

\section{Spectromicroscopy for biomedical specimens}

$\mathrm{X}$-ray spectromicroscopy is a powerful imaging tool to obtain molecular distribution in biological specimens. The scanning transmission X-ray microscope (STXM) has been frequently used for this purpose. The illustrated system is inserted in Fig. 2, i.e., specimen is scanned on a monochromatic X-rays focused by a zone plate and transmitted X-rays are detected. The pioneering work by Ade et al. was conducted for DNA mapping in a chromosome using the difference in X-ray absorption near edge structure (XANES) profiles between DNA and protein at the C $K$ absorption edge [6]. The same methodology was successfully applied to DNA distribution in a bull sperm [7]. We proposed that the use of XANES at the N $K$ absorption edge would be better for the mapping of DNA due to the presence of a unique $\pi *$ peak [8]. Using newly built STXM installed at UVSOR in Japan [9], we have obtained the N $K$ edge spectra and examined DNA and protein distribution in cell nuclei during the progression of apoptosis. Apoptosis is well known as a programmed cell death and is characterized by remarkable morphological changes in a cell nucleus. Tone et al. has extensively studied the progression of the morphological changes by labelling DNA with fluorescent dye for isolated nuclei using optical microscope, and proposed that the changes in a nucleus proceeded via three stages [10]. We intended to follow the process with a spatial resolution around $100 \mathrm{~nm}$.


Fig. 2. Relative distribution of DNA and protein in dried apoptotic nucleus in the "stage 1". (a) Horizontal line profile of DNA distribution in part (b), (b) DNA distribution, (c) protein distribution, (d) horizontal line profile of protein distribution in part (c). Insert figure shows typical optical layout of STXM. Scale bar corresponds to $1 \mu \mathrm{m}$.

Although the research is in progress, the preliminary results for the relative distributions of DNA and nuclear protein such as histone in dried nucleus at stage 1 are shown in Fig. 2. DNA and protein were found to be preferentially located in the peripheral region leading to the formation of a ring structure. The horizontal line profiles across the nucleus (part (a) and (d) in Fig. 2) indicated 

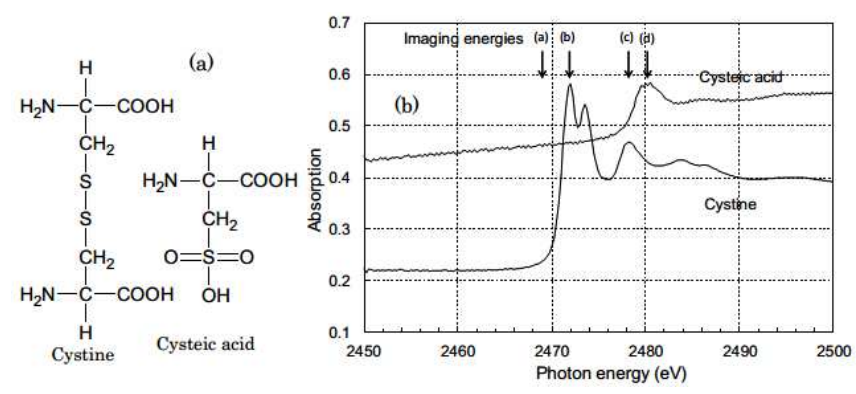

Fig. 3. Chemical structures of sulfur containing amino acids and their XANES at the S $K$ absorption edge. (a) Chemical structures of cystine and its oxidation product, cysteic acid. (b) XANES of cystine and cysteic acid. Imaging energies (a) to (d) indicated by arrows were used to obtain maps of cystine and cysteic acid by a subtraction method.

that DNA content was nearly constant with slight increase in the peripheral region, while protein was more accumulated in the peripheral region, which suggests that DNA may be present without histone in the inner region. The meaning of this observation is left for future research. Molecular images of all stages in the process of apoptosis are referred to the proceedings of the International Conference on X-Ray Microscopy in 2014 [11].

Another application is an imaging of oxidation state of cystine in human hair, which was conducted at relatively high X-ray energy region near hard X-rays including the $\mathrm{S} K$ edge $(2.47 \mathrm{keV})$ and the Ca $K$ edge $(4.04 \mathrm{keV})$. Human hair always undergoes various outer oxidative stimuli such as bleach treatment and exposure to ultraviolet radiation. In order to provide the efficient protection of hair against these oxidative stresses, the information about the distribution of oxidative damage is definitely useful. Human hair is primarily composed of keratin protein characterized by high content of sulfurcontaining amino acid, cystine. Exposure to oxidative stress induces cysteic acid, an oxidative form of cystine (Fig. 3a). Since these two forms of amino acids have different XANES peaks at the $\mathrm{S} K$ absorption edge as shown in Fig. 3b [12], we could obtain the distribution of cysteic acid (oxidation map) by the subtraction of images taken at the energy (c) and (d) [13]. For a cystine map, the subtraction between energy (a) and (b) was performed. The images were taken by a contact X-ray microscopy with a high spatial resolution two-dimensional detector, an electronic zooming tube at a spatial resolution of about $0.5 \mu \mathrm{m}$ over the energies used at the Photon Factory, Japan.

Figure 4 indicates the influence of bleach treatment at a beauty parlor on the oxidation in female hair. Hair without any bleach treatment has little oxidation as seen from the cysteic acid map, while with increasing times of bleach treatment the oxidation was evidently increased over the whole area of the hair. The right column pictures show Ca distribution. Accumulation of calcium

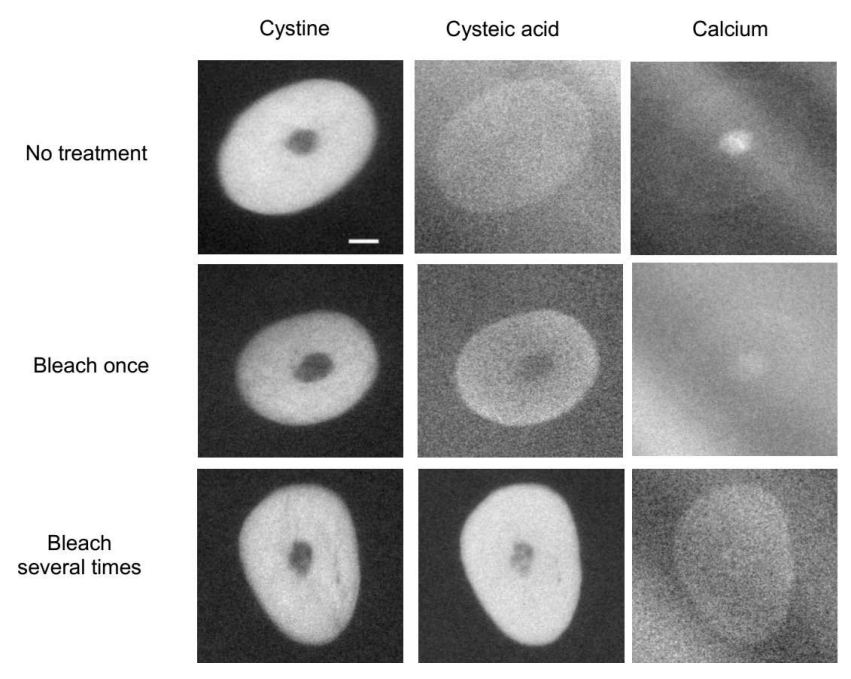

Fig. 4. Distributions of oxidation product and $\mathrm{Ca}$ in human hair with bleach treatment at a beauty parlor. Dried cross-sectionally sliced thin section with a thickness of about $20 \mu \mathrm{m}$ of hair was observed by an X-ray contact microscopy. Cystine and cysteic acid maps were shown for hair specimens treated with different degree of bleaching. Ca maps are displayed for comparison. Scale bar corresponds to $20 \mu \mathrm{m}$.

has been reported to have a close correlation to oxidative damage [14], and recent interesting observations suggested the possible correlation between $\mathrm{Ca}$ content in hair and the incidence of breast cancer [15]. Based on these preceding observations we measured the localization of $\mathrm{Ca}$ to compare with an oxidation map at high spatial resolution. The calcium map was obtained at the Ca $K$ edge using XANES profiles of Ca compounds. Figure 4 shows distributions of cystine, cysteic acid and $\mathrm{Ca}$ to compare bleached hair with normal unbleached hair. Cystine maps reflect the thickness of hair specimens, that is, the specimen with no treatment has largest thickness compared with other specimens. Considering the thickness of specimens, increasing bleach treatment increased Ca content as well as oxidation in the regions of cuticle, the peripheral part of hair, and cortex, the inner part of hair, which was in accord with the previous reports except for the central structure called medulla. Ca content in medulla is not likely to depend on the degree of oxidation. For example, Ca content was significant even in untreated hair. The Ca distributions were also confirmed by $\mathrm{X}$-ray fluorescence mapping using scanning microbeam X-rays at the Photon Factory (data not shown). The experiments using hair specimens with bleach treatment in our laboratory give similar results [16]. These findings indicate that in determining the Ca contents in hair the detailed distribution of calcium inside hair should be taken into account.

The present results demonstrated the potential of the soft X-ray spectromicroscopy to provide a high spatial resolution map of a specific molecule which cannot be ob- 
tained by other imaging technique. However, note that these experiments have been done with dry specimens. The method should be extended to the observation in hydrated specimens using rapid freezing method for the advancement of soft X-ray spectromicroscopy in biomedical applications.

\section{Acknowledgments}

The work was performed at the Photon Factory under the application number 2008G536, 2010G542, and 2012 G626 and also a part of this work was conducted in UVSOR, supported by Nanotechnology Platform Program (Molecule and Material Synthesis) of the Ministry of Education, Culture, Sports, Science and Technology (MEXT), Japan. This work was partly supported by the Japan Society for the Promotion of Science (JSPS) KAKENHI, Grant-in-Aid for Scientific Research (C) (25390128)

\section{References}

[1] D. Attwood, Soft X-rays and Extreme Ultraviolet: Principles and Applications, Cambridge University Press, New York 1999.

[2] A. Ito, K. Shinohara, Cell Struct. Funct. 17, 209 (1992).

[3] D. Sayre, J. Kirz, R. Feder, D.M. Kim, E. Spiller, Ultramicroscopy 2, 337 (1977).

[4] M. Kado, M. Kishimoto, S. Tamotsu, K. Yasuda, K. Shinohara, J. Phys. Conf. Ser. 463, 012056 (2013).
[5] G. McDermott, M.A. Le Gros, C.G. Knoechel, M. Uchida, C.A. Larabell, Trends Cell Biol. 19, 587 (2009).

[6] H. Ade, X. Zhang, S. Cameron, C. Costello, J. Kirz, S. Willams, Science 258, 972 (1992).

[7] X. Zhang, R. Balhorn, J. Mazrimas, J. Kirz, J. Struct. Biol. 116, 335 (1996).

[8] K. Shinohara, A. Ito, K. Kobayashi, in: X-Ray Microscopy and Spectromicroscopy, Eds. J. Thieme, G. Schmahl, E. Umbach, D. Rudolph, Springer, Heidelberg 1998, p. III-157.

[9] T. Ohigashi, H. Arai, T. Araki, N. Kondo, E. Shigemasa, A. Ito, N. Kosugi, M. Katoh, J. Phys. Conf. Ser. 463, 012006 (2013).

[10] S. Toné, K. Sugimoto, K. Tanda, T. Suda, K. Uehira, H. Kanouchi, K. Samejima, Y. Minatogawa, W.C. Earnshaw, Exp. Cell Res. 313, 3635 (2007).

[11] T. Ohigashi, A. Ito, K. Shinohara, S. Tone, M. Kado, Y. Inagaki, Y.F. Wang, N. Kosugi, AIP Conf. Proc. 1696, 020027 (2016).

[12] A. Ito, T. Inoue, K. Takehara, N. Shimizu, Y. Kitajima, K. Shinohara, J. X-ray Sci. Technol. 19, 249 (2011).

[13] T. Inoue, K. Takehara, N. Shimizu, Y. Kitajima, K. Shinohara, A. Ito, J. X-ray Sci. Technol. 19, 313 (2011).

[14] K.E. Smart, M. Kilburn, M. Schroeder, B.G. Martin, C. Hawes, J.M. Marsh, C.R. Grovenor, J. Cosmet. Sci. 60, 337 (2009).

[15] J. Chikawa, K. Yamada, T. Akimoto, H. Sakurai, H. Yasui, H. Yamamoto, S. Okabe, M. Ebara, J. Xray Sci. Technol. 15, 109 (2007).

[16] A. Ito, T. Inoue, T. Kawai, Y. Taki, S. Inoue, T. Shimizu, K. Shinohara, AIP Conf. Proc. 1696 , 020021 (2016). 